Je me suis servi dans ce but de la méthode de Nencki et Duclaux. Avant tout, on a déterminé la quantité produite d'acide lactique en mesurant la chaux dissoute. On a trouvé que la quantité trouvée d'acide lactique correspond parfaitement à la quantité calculée. Puis on a distillé pour chercher les produits volatils. On n'a obtenu ni alcool ni éther et ni acides volatils.

Toutes ces recherches montrent que le $B$. caucasicum ne forme pas d'autres produits que de l'acide lactique.

\title{
ANALYSE DU LAIT CONDENSÉ SUCRÉ
}

\section{par F. RICHARD.}

L'analyse de ce produit présente de sérieuses difficultés en raison de sa grande viscosité, et le chimiste, s’il n'est pas spécialisé en la matière, se trouve en présence d'un problème embarrassant. S'il consulte les auteurs, il s'aperçoit qu'ils sont d'une rare sobriété de renseignements sur les procédés à employer pour ce cas spécial. Après de nombreux tâtonnements, je me suis arrêté à la méthode que je vais décrire, et je crois bien faire en indiquant les moindres détails des opérations qui doivent conduire à d'indiscutables résultats.

En général, on vous demande de déterminer la densité à $15^{\circ} \mathrm{C}$., le pourcentage en eau, matière grasse, et sucre cristallisable (saccharose). Il est rare qu'on soit appelé à doser le lactose, la matière azotée et la matière minérale.

Etant donné le peu de fluidité du L. C. (lait condensé), il est impossible d'en prendre directement le poids spécifique, soit au densimètre, soit à la balance de Westphal ; pour la même raison, le dosage de l'eau devient une opération incertaine, car, dès qu'on veut pousser un peu 'la température au delà de $100^{\circ} \mathrm{C}$., l'extrait se caramélise avec la plus grande facilité. Pour réussir l'analyse, il faut avant tout, préparer des solutions aqueuses soigneusement dosées; il est recommandable de faire toutes les pesées du L. C. dès que la boîte est ouverte, car il arrive souvent que la surface de certains échantillons se couvre d'une croûte dure au bout de quelque temps de contact avec l'air.

A l'ouverture de la boîte, on brasse bien la masse au moyen d'une forte baguette de verre, surtout si l'on affaire à du L. C. non écrémé, et immédiatement on procède aux :

Opérations préliminaires: $1^{\circ}$ Peser $50 \mathrm{gr}$. de L. C. dans une capsule tarée, y ajouter environ 20 c.c. d'eau distillée et agiter prudemment avec une petite baguette de verre pour délayer la masse, on verse le mélange dans un petit entonnoir placé sur un ballon jaugé de 100 c.c., on rince capsule et entonnoir par petites portions d'eau, on amène le mélange à la température de $15^{\circ} \mathrm{C}$., et complète le volume à 100 c.c., on secoue bien de temps en temps, pour avoir un mélange parfaitement homogène. C'est la solution $A$. 
$2^{\circ}$ Peser 33 gr. 33 L. C. et faire comme ci-dessús un volumede 100 c. c. à $15^{\circ} \mathrm{C}$. C'est la solution $B$.

Densité. Les solutions A et B étant à $15^{\circ} \mathrm{C}$., on en détermine la densité ; l'emploi de la balance de Westphal est préférable à celui des aréomètres qui sont rarement rigoureusement exacts.

Soit: D, la densité du L. C.

P, le poids de L. C. pour faire la solution de 100 c.c.

d, la densité trouvée pour cette solution.

$$
\begin{aligned}
& D=\frac{P}{100-(100 d-P)} \text { d'où } d=\frac{P\left(100-\frac{P}{D}\right)}{100} \\
& P=\frac{100 D(d-1)}{D-1}
\end{aligned}
$$

Exemple : la solution A donne une densité $d=1.117$

$\mathrm{D}=\frac{50}{100-(111.7-50)}=\frac{50}{38.3}=1.305$

On contrôle la densité d' de la solution B.

Cette densité sera $\mathrm{d}=\frac{33.33\left(100-\frac{33.33}{1.305}\right)}{100}=1.077$

S'il y avait discordance entre les deux densités, c'est que les solutions n'auraient pas été suffisamment secouées, et que le mélange ne serait pas assez homogène. Quoi qu'il en soit, si l'écart n'est pas trop grand, on prendra la moyenne.

Dosage de 1'eau. Dans une eapsule plate, soigneusement tarée, on pèse 2 grammes de L. C., on y ajoute 1 à 2 c.c. d'eau et porte à l'étuve chauffée à $100^{\circ} \mathrm{C}$.; au bout de 10 minutes, quand la capsule est chaude on remue le mélange en le roulant en tous sens, de manière à avoir une couche mince sur toute la surface. Si on ne prend pas cette précaution, la matière ne s'étale pas et au bout de 20 heures on n'aurait pas encore un produit sec.

II faut avoir soin de ne pas dépasser $100^{\circ} \mathrm{C}$, carl'extrait se caramélise très facilement et le dosage deviendrait incertain.

Lorsque la matière parait sèche, généralement au bout de 2 heures, on pèse et note le poids, puis on tâte avec un stylet ou un canif s'il n'y a plus de parties molles; dans ce cas, on les lacère en tous sens avec la lame et on remet à l'étuve pendant une heure, on repèse, et continue à sécher jusqu'à poids constant.

Je contrôle toujours le dosage de l'eau par un essai parallèle. Pour cela je pèse $5 \mathrm{cc}$. de la solution $\mathrm{A}$ dans un large couvercle en fer blanc bien plat; on ne peut se contenter de mesurer 5 ec de la solution. A., il faut peser, en tenant compte de la densité $d$, c'est-à-dire $5 \times d$, dans 
l'exemple que j'ai cité $\mathrm{d}=1.117$; on pèse done $5 \mathrm{gr} .585$, qui représentent 2 gr. 50 de L. C. et on sèche comme ci-dessus.

En opérant de cette manière, on est certain de ses résultats.

Nous avons trouvé :

10 Pourles 2 gr., perte de poids 0 gr. 514 , soit: eau $=\frac{0.514 \times 100}{2}=25.7$.

20. Pour les 2 gr. 50 , perte 0 gr. 645 , soit: eau $=\frac{0.645 \times 100}{2.5}=25.8$

Dosage de la matière grasse. Le procédé GERBER est très commode pour ce dosage, et afin d'avoir tous ses apaisements, on opère sur les solutions $\mathrm{A}$ et $\mathrm{B}$, on multiplie le résultat de $\mathrm{A}$ par 2 , et celui de $\mathrm{B}$ par 3 ; au lieu de mesurer les solutions à la pipette, je préfère emplover une burette graduée à robinet, ear la pipette donne toujours des résultats trop faibles; à défaut de GerBer, on peut recourir au procédé GotTlineBRösE.

J'ai trouvé pour A: $3.6 \times 2=7.2$, et pour $B: 2.4 \times 3=7.2$.

Dosage du saccharose. Le principe est le suivant: Déterminer l'action réductrice du L. C. sur la liqueur de Fehling, avant et après inversion.

Dans ce but, il y a deux nouvelles solutions à préparer :

$1^{\circ}$ On pèse 10 c.c. de la solution $A$, e'est-à-dire $10 \times d$, dans notre exemple $11 \mathrm{gr} .17$; on verse dans un ballon jaugé de 100 c.e., rince à l'eau distillée, et avant de compléter le volume à 100 , on y laisse tomber deux gouttes d'acide acétique, on agite, laisse reposer, et lorsque le coagulum s'est bien formé, on filtre ; on obtient une liqueur parfaitement claire, c'est la solution M dont 100 c.c. représentent 5 gr. L. C.

$2^{\circ}$ On prélève 50 c.c. de la solution $\mathrm{M}$, verse dans un vase de Ferlin, y ajoute 10 gouttes d'acide ehlorhydrique et fait bouillir pendant 10 minutes pour transformer la saccharose en glucose, on neutralise au carbonate de soude, laisse refroidir, et verse dans un ballon jaugé de 100 c.c., rince et complète à 100, c'est la solution $\mathrm{N}$ dont 100 c.c. représentent $2 \mathrm{gr}$. $50 \mathrm{~L}$. C.

a) Détermination de l'action réductrice de la solution M (avant inversion): Dans un ballon de 400 , on met 40 c.c. d'eau, un peu de pierre ponce et 10 c.c. de liqueur de Fehling dont 1 c.c. est réduit par 0 gr. 005 de glucose; on fait bouillir et on y laisse tomber d'une burette graduée-la solution M, jusqu'à complète réduction; on verse d'emblée 7 c.c. et laisse bouillir pendant une minute, puis on continue avec prudence jusqu'au point final ; après chaque addition, on fait bouillir, on place ensuite le ballon sur un papier blane et laisse déposer l'oxydule de euivre pour juger de la teinte de la solution. Dans le cas de l'échantillon pris comme exemple, il m'a fallu 11 c.c. 6 de solution M pour obtenir la réduction complète de la liqueur de Fehling. La quantité 
de glucose équivalente pour 109 L. C. $=\frac{100}{11.6}=8,62$

b) Détermination de l'action réductrice de la solution $\mathrm{N}$ (après inversion) : On opère comme ci-dessus sur 10 c.e. de liqueur de FentING, mais ici, le sucre étant interverti, il faudra beaucoup moins de solution pour produire la réduction complète. En conséquence, on ne mettra d'emblée que 3 c.e. de solution $\mathrm{N}$ et on avancera avec une extrême prudence; il m'a fallu 3 c.c. 8 , la quantité de glucose équivalente pour $100 \mathrm{I}$. C. $=\frac{200}{3.8}=52.6$.

Le glucose dû au sucre interverti est donc :

$52.6-8.6=44.0$, or 1 glucose $=0.95$ saceharose ; donc $44 \times 0.95=41.8 \%$ saccharose.

Pour ces titrages il faut opérer avec beaucoup de précision, car une différence d'un dixième de c.c. en plus ou en moins change totalement le résultat; ainsi pour 3 c.c. 7 on aurait $\frac{200}{3.7}=54.0$ et pour 3.9 on aurait $\frac{200}{3.9}=51.3$.

C'est pourquoi il est bon de faire d'abord un titrage approximatif, pour connaître la quantité de liquide qu'on peut verser d'emblée dans la liqueur de Fenting, et n'avancer ensuite que par deux gouttes à la fois.

Préparation de la liqueur de Fehling. Il faut faire deux solutions séparées et ne les mélanger à parties égales qu'au fur et à mesure de ses besoins, car la liqueur toute préparée ne se conserve que pendant très peu de temps.

$1^{\circ}$ Dissoudre dans de l'eau distillée $34 \mathrm{gr}$. 64 de sulfate de cuivre pur en beaux cristaux non effleuris et secs, et porter le volume à 500 c.c.

$2^{\circ}$ Dissoudre $173 \mathrm{gr}$. de sel de Seignette (tartrate double de potasse et de soude) dans une solution de soude caustique d'une densité de 1.12, de manière à obtenir 500 c.́c. ; on peut remplacer le sel de Seignette par 93 gr. 5 de crème de tartre.

Pour s'exercer à titrer les solutions de glucose, on fait dissoudre 0 gr. 95 dè sucre candi blanc bien cristallisé dans 50 à 60 c.c. d'eau, on y ajoute 1 c.c. d'acide chlorhydrique et fait bouillir pendant 10 minutes, neutralise au carbonate de soude, laisse refroidir et complète le volume à 100 . Il faut 5 c.c. de cette solution pour réduire 10 c.c. liqueur de FEHLING.

Dosage du lactose. Nous avons vu que la solution M, c'est-à-dire avant inversion, réduisait la liqueur de FEHLING; cette réduction est due au lactose. 
Tous les auteurs impriment que pour avoir le lactose correspondant à la quantité de glucose trouvée, il suffit de multiplier celle-ci par 1.27; je regrette pour les auteurs de devoir leur dire que c'est là une erreur qui s'imprime et se transmet de génération en génération depuis plus de 60 ans. En effet, ayant été appelé à faire des analyses complètes de lait condensé, je me suis aperçu qu'en fin d'analyse, je n'arrivais pas à mon compte, en additionnant tous les résultats. C'est ce qui -m'a amené à contrôler le facteur du sucre de lait. Pour réduire 10 c.c. de liqueur de Frhuing, il me fallait 7 e.c. 9 d'une solution de lactose à $1 \%$, c'est-àdire que 0 gr, 79 lactose correspondent à 0 gr. 50 glucose, d'où 1 glucose = 1.58 lactose. Le multiplicateuir est donc 1.58 et non 1.27. Dans l'exemple d'analyse que nous avons pris, nous avons vu qu'il fallait 11 c.c. 6 de solution M pour réduire 10 c.c. de liqueur de FeHLING; ce qui correspond à $8.6 \%$ glucose $; 8.6 \times 1.58=13.6$ lactose .

Matière azotée. Il est très rare qu'on procède à ce dosage, on se contente généralement d'inscrire comme matière azotée la différence entre 100 et le total des autres éléments trouvés. On n'obtient ainsi qu'un chiffre approximatif, car le lait contient en petite quantité des éléments peu déterminés, tels des citrates qui s'ajoutent à la matière azotée. On peut aussi calculer la teneur en matière albuminoïde en se basant sur celle en sucre de lait; soit S. L. cette teneur: $\frac{\text { SI } \times 3.6}{5.2}=$ matière azotée.

Le moyen le plus sûr c'est de déterminer l'azote par le procédé de Kjedahl ; on opère sur 5 gr. L. C. ; l'opération est longue et pénible, et pour finir, la distillation de l'ammoniaque est rendue trés délicate à cause de la mousse abondante, due au savon provenant de l'action de la soude sur le beurre. J'avais obtenu $1.47 \%$ azote. $1.47 \times 6.3=9.3$ matière azotée.

Matières minérales. On pèse $5 \mathrm{gr}$. L, C. soit sur une capsule de platine soit dans un creuset de porcelaine, on sèche à l'étuve en chauffant au-delà de $100^{\circ} \mathrm{C}$, puis on calcine au rouge très sombre, pour ne pas volatiliser les chlorures, on pèse et multiplie le résultat par 20.

J'avais trouvé $0.094 \times 20=1.88 \%$.

En récapitulant tous les résultats, nous obtenons :

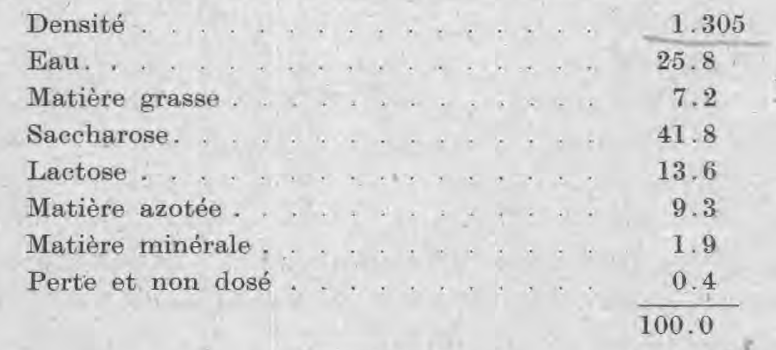

